\title{
Repairing MIP infeasibility through Local Branching
}

\author{
Matteo Fischetti* Andrea Lodi ${ }^{\circ} \dagger$ \\ * DEI, University of Padova, Via Gradenigo 6A - 35131 Padova - Italy \\ 'T.J. Watson Research Center, IBM, Yorktown Heights, NY 10598, USA \\ $\dagger$ DEIS, University of Bologna, Viale Risorgimento 2 - 40136 Bologna - Italy \\ e-mail: \{matteo.fischetti@unipd.it, alodi@us.ibm.com\}
}

February 16, 2004; Revised September 3, 2005

\begin{abstract}
Finding a feasible solution to a generic Mixed-Integer Program (MIP) is often a very difficult task. Recently, two heuristic approaches called Feasibility Pump and Local Branching have been proposed to address the problem of finding a feasible solution and improving it, respectively. In this paper we introduce and analyze computationally a hybrid algorithm that uses the feasibility pump method to provide, at very low computational cost, an initial (possibly infeasible) solution to the local branching procedure which can indeed work also with infeasible solutions. The overall procedure is reminiscent of Phase I of the two phase simplex algorithm, in which the original LP is augmented with artificial variables that make a known infeasible starting solution feasible and then the augmented model is solved to iteratively reduce that infeasibility by driving the values of the artificial variables to zero. As such, our approach can also to used to find (heuristically) a minimum-cardinality set of constraints whose removal converts an infeasible MIP into a feasible one-a very important piece of information in the analysis of infeasible MIP models.
\end{abstract}

\section{Introduction}

In this paper, we consider the problem of finding a feasible solution to a generic Mixed-Integer Program (MIP) with 0-1 variables of the form:

$$
\begin{array}{ll}
(P) \quad & \min c^{T} x \\
& \text { s.t. } \\
& A x \geq b, \\
& x_{j} \in\{0,1\}, \forall j \in \mathcal{B} \neq \emptyset, \\
& x_{j} \geq 0, \text { integer, } \forall j \in \mathcal{G}, \\
& x_{j} \geq 0, \forall j \in \mathcal{C},
\end{array}
$$

where $A$ is a $m \times n$ input matrix, and $b$ and $c$ are input vectors of dimension $m$ and $n$, respectively. Here, the variable index set $\mathcal{N}:=\{1, \ldots, n\}$ is partitioned into $(\mathcal{B}, \mathcal{G}, \mathcal{C})$, where $\mathcal{B} \neq \emptyset$ is the index set of the $0-1$ variables, while the possibly empty sets $\mathcal{G}$ and $\mathcal{C}$ index the general integer and the continuous variables, respectively. Note that we assume the existence of 0-1 variables, as one of the components of the method we actually implemented (namely, the local branching heuristic) is based on this assumption; our approach can however be extended to get rid off this limitation, as outlined in the concluding remarks of [9]. Also note 
that constraints (3), though stated as inequalities, can involve equalities as well. Let $\mathcal{I}:=\mathcal{B} \cup \mathcal{G}$ denote the index set of all integer-constrained variables.

Heuristics for general-purpose MIPs include [3], [4], [5], [7], [11], [13], [14], [15],[16], [17], [19], [20], [21], and [24], among others. Recently, we proposed in [9] a heuristic approach, called Local Branching (LB), to improve the quality of a given feasible solution. This method, as well as other refining heuristics (including the recently-proposed RINS approach [7]), requires the availability of a starting feasible solution, which is an issue for some difficult MIPs. This topic was investigated by Fischetti, Glover and Lodi [10], who introduced the so-called Feasibility Pump (FP) scheme for finding a feasible (or, at least, an "almost feasible") solution to general MIPs through a clever sequence of roundings.

In the present paper, we analyze computationally a simple variant of the original LB method that allows one to deal with infeasible reference solutions, such as those returned by the FP method. Our approach is to start with an "almost feasible" reference solution $\bar{x}$, as available at small computational cost through the FP method. We then relax the MIP model by introducing for each violated constraint: (i) an artificial continuous variable in the constraint itself, (ii) a binary (also artificial) variable, and (iii) a constraint stating that, if the artificial variable has to be used to make the constraint satisfied, then the binary variable must be set to 1. Finally, the objective function is replaced, in the spirit of the first phase of the primal simplex algorithm, by the sum of the artificial binary variables. The initial solution turns out now to be feasible for the relaxed model and its value coincides with the number of initial violated constraints. We then apply the standard LB framework to reduce the value of the objective function, i.e., the number of infeasibilities and a solution of value 0 turns out to be feasible for the initial problem. Note that, although a continuous artificial variable for each violated constraint could be enough, binary variables are better exploited by LB as it will be clear from Section 2 and discussed in detail in Section 3.

Our approach also produces, as a byproduct, a small-cardinality set of constraints whose relaxation (removal) converts a given MIP into a feasible one-a very important piece of information in the analysis of infeasible MIPs. In other words, our method can be viewed as a tool for repairing infeasible MIP models, and not just as a heuristic for repairing infeasible MIP solutions. This is in the spirit of the widely-studied approaches to find maximum feasible (or minimum infeasible) subsystems of LP models, as addressed e.g. in $[2,6,12]$, but applies to MIP models - hence it may be quite useful in practice.

The paper is organized as follows. In Section 2 we review the LB and FP methods. In Section 3 we describe the LB extension we propose to deal with infeasible reference solutions. Computational results are presented in Section 4, where we compare the LB performance with that of the commercial software ILOG-Cplex on two sets of hard 0-1 MIPs, specifically 44 problems taken from MIPLIB 2003 library [1] and 39 additional instances already considered in [10].

\section{Local Branching and Feasibility Pump}

We next review the LB and FP methods; the reader is referred to [9] and [10] for more details.

\section{Local Branching}

The Local Branching approach works as follows. Suppose a feasible reference solution $\bar{x}$ of $(P)$ is given, and one aims at finding an improved solution that is "not too far" from $\bar{x}$. Let $\bar{S}:=\left\{j \in \mathcal{B}: \bar{x}_{j}=1\right\}$ denote the binary support of $\bar{x}$. For a given positive integer parameter $k$, we define the $k$-OPT neighborhood $\mathcal{N}(\bar{x}, k)$ of $\bar{x}$ as the set of the feasible solutions of $(P)$ satisfying the additional local branching constraint:

$$
\Delta(x, \bar{x}):=\sum_{j \in \bar{S}}\left(1-x_{j}\right)+\sum_{j \in \mathcal{B} \backslash \bar{S}} x_{j} \leq k,
$$

where the two terms in the left-hand side count the number of binary variables flipping their value (with respect to $\bar{x}$ ) either from 1 to 0 or from 0 to 1 , respectively. As its name suggests, the local branching constraint (7) can be used as a branching criterion within an enumerative scheme for $(P)$. Indeed, given the 
incumbent solution $\bar{x}$, the solution space associated with the current branching node can be partitioned by means of the disjunction

$$
\Delta(x, \bar{x}) \leq k \quad(\text { left branch }) \text { or } \Delta(x, \bar{x}) \geq k+1 \quad \text { (right branch) }
$$

where the neighborhood-size parameter $k$ is chosen so as make neighborhood $\mathcal{N}(\bar{x}, k)$ "sufficiently small" to be optimized within short computing time, but still "large enough" to likely contain better solutions than $\bar{x}$ (typically, $k=10$ or $k=20$ ).

In [9], we investigated the use of a general-purpose MIP solver as a black-box "tactical" tool to explore effectively suitable solution subspaces defined and controlled at a "strategic" level by a simple external branching framework. The procedure is in the spirit of well-known local search metaheuristics, but the neighborhoods are obtained through the introduction in the MIP model of the local branching constraints (7). This allows one to work within a perfectly general MIP framework, and to take advantage of the impressive research and implementation effort that nowadays are devoted to the design of MIP solvers. The new solution strategy is exact in nature, though it is designed to improve the heuristic behavior of the MIP solver at hand. It alternates high-level strategic branchings to define solution neighborhoods, and low-level tactical branchings (performed within the MIP solver) to explore them. The result can then be viewed as a two-level branching strategy aimed at favoring early updatings of the incumbent solution, hence producing improved solutions at early stages of the computation. The computational results reported in [9] show the effectiveness of the LB approach. These have also been confirmed by the recent works of Hansen, Mladenovíc and Urosevíc [16] (where LB is used within a Variable Neighborhood Search metaheuristic [23]) and of Fischetti, Polo and Scantamburlo (where MIPs with a special structure are investigated).

\section{Feasibility Pump}

Let $P_{L}:=\left\{x \in \Re^{n}: A x \geq b\right\}$ denote the polyhedron associated with the LP relaxation of the given MIP, and assume without loss of generality that system $A x \geq b$ includes the variable bounds

$$
l_{j} \leq x_{j} \leq u_{j}, \quad \forall j \in \mathcal{I},
$$

where $l_{j}=0$ and $u_{j}=1$ for all $j \in \mathcal{B}$. With a little abuse of notation, we say that a point $x$ is integer if $x_{j} \in Z^{n}$ for all $j \in \mathcal{I}$ (no matter the value of the other components). Analogously, the rounding $\tilde{x}$ of a given $x$ is obtained by setting $\tilde{x}_{j}:=\left[x_{j}\right]$ if $j \in \mathcal{I}$ and $\tilde{x}_{j}:=x_{j}$ otherwise, where [.] represents scalar rounding to the nearest integer. The $\left(L_{1}\right.$-norm) distance between a generic point $x \in P_{L}$ and a given integer vector $\tilde{x}$ is defined as

$$
\Phi(x, \tilde{x})=\sum_{j \in \mathcal{I}}\left|x_{j}-\tilde{x}_{j}\right|,
$$

(notice that the continuous variables $x_{j}, j \notin \mathcal{I}$, if any, are immaterial) and can be modeled as

$$
\Phi(x, \tilde{x}):=\sum_{j \in \mathcal{I}: \tilde{x}_{j}=l_{j}}\left(x_{j}-l_{j}\right)+\sum_{j \in \mathcal{I}: \tilde{x}_{j}=u_{j}}\left(u_{j}-x_{j}\right)+\sum_{j \in \mathcal{I}: l_{j}<\tilde{x}_{j}<u_{j}}\left(x_{j}^{+}+x_{j}^{-}\right),
$$

where the additional variables $x_{j}^{+}$and $x_{j}^{-}$require the introduction into the MIP model of the additional constraints:

$$
x_{j}=\tilde{x}_{j}+x_{j}^{+}-x_{j}^{-}, \quad x_{j}^{+} \geq 0, x_{j}^{-} \geq 0, \quad \forall j \in \mathcal{I}: l_{j}<\tilde{x}_{j}<u_{j} .
$$

It then follows that the closest point $x^{*} \in P_{L}$ to $\tilde{x}$ can easily be determined by solving the LP

$$
\min \{\Phi(x, \tilde{x}): A x \geq b\} .
$$

If $\Phi\left(x^{*}, \tilde{x}\right)=0$, then $x_{j}^{*}\left(=\tilde{x}_{j}\right)$ is integer for all $j \in \mathcal{I}$, so $x^{*}$ is a feasible MIP solution. Conversely, given a point $x^{*} \in P_{L}$, the integer point $\tilde{x}$ closest to $x^{*}$ is easily determined by just rounding $x^{*}$. 
The FP heuristic works with a pair of points $\left(x^{*}, \tilde{x}\right)$ with $x^{*} \in P_{L}$ and $\tilde{x}$ integer, that are iteratively updated with the aim of reducing as much as possible their distance $\Phi\left(x^{*}, \tilde{x}\right)$. To be more specific, one starts with any $x^{*} \in P_{L}$, and initializes a (typically infeasible) integer $\tilde{x}$ as the rounding of $x^{*}$. At each FP iteration, called a pumping cycle, $\tilde{x}$ is fixed and one finds through linear programming the point $x^{*} \in P_{L}$ which is as close as possible to $\tilde{x}$. If $\Phi\left(x^{*}, \tilde{x}\right)=0$, then $x^{*}$ is a MIP feasible solution, and the heuristic stops. Otherwise, $\tilde{x}$ is replaced by the rounding of $x^{*}$ so as to further reduce $\Phi\left(x^{*}, \tilde{x}\right)$, and the process is iterated.

The basic FP scheme above tends to stop prematurely due to stalling issues. This happens whenever $\Phi\left(x^{*}, \tilde{x}\right)>0$ is not reduced when replacing $\tilde{x}$ by the rounding of $x^{*}$, meaning that all the integer-constrained components of $\tilde{x}$ would stay unchanged in this iteration. In the original FP approach [10], this situation is dealt with by heuristically choosing a few components $\tilde{x}_{j}$ to be modified, even if this operation increases the current value of $\Phi\left(x^{*}, \tilde{x}\right)$. A different approach, to be elaborated in the next section, is to switch to a method based on enumeration, in the attempt to explore a small neighborhood of the current "almost feasible" $\tilde{x}$ (that typically has a very small distance $\Phi\left(x^{*}, \tilde{x}\right)$ from $P_{L}$ ).

\section{$3 \quad$ LB with Infeasible Reference Solutions}

The basic idea of the method presented in this section is that the LB algorithm does not necessarily need to start with a feasible solution - a partially feasible one can be a valid warm start for the method. Indeed, by relaxing the model in a suitable way, it is always possible to consider any infeasible solution, say $\hat{x}$, to be "feasible", and penalize its cost so the LB heuristic can drive it to feasibility.

The most natural way to implement this idea is to add a continuous artificial variable for each constraint violated by $\hat{x}$, and then penalize the use of such variables in the objective function by means of a very large cost $M$. We tested this approach and found it performs reasonably well on most of the problems. However, it has the drawback that finding a proper value for $M$ may not be easy in practice. Indeed, for a relevant set of problems in the MIPLIB 2003 [1] collection, the value of the objective function is so large that it is difficult to define a value for $M$ that makes any infeasible solution worse than any feasible one. Moreover, the way the LB method works suggests the use of a more combinatorial framework.

Let $T$ be the set of the indices of the constraints $a_{i}^{T} x \geq b_{i}$ that are violated by $\hat{x}$. For each $i \in T$, we relax the original constraint $a_{i}^{T} x \geq b_{i}$ into $a_{i}^{T} x+\delta_{i} y_{i} \geq b_{i}$, where $\delta_{i}:=b_{i}-a_{i}^{T} \hat{x}$ is the positive amount of violation computed with respect to $\hat{x}$, and $y_{i}$ is a binary artificial variable attaining value 1 for each constraint violated by $\hat{x}$. Finally, we replace the original objective function $c^{T} x$ by $\sum_{i \in T} y_{i}$, so as to count the number of violated constraints. It has to be noted that the set of binary variables in the relaxed model is $\mathcal{B} \cup \mathcal{Y}$, where $\mathcal{Y}:=\left\{y_{i}: i \in T\right\}$, hence the structure of the relaxation turns out to be particularly suited for the LB approach, where the local branching constraint affects precisely the binary variables (including the artificial ones).

An obvious drawback of the method above is that the original objective function is completely disregarded, thus the feasible solution obtained can be arbitrarily bad. A way of avoiding this situation could be to put a term in the artificial objective function that takes the original costs into account. However, a proper balancing of the two contributions (original cost and infeasibility penalty) may not be easy to achieve. As a matter of fact, the outcome of a preliminary computational study is that a better overall performance is obtained by using the artificial objective function (alone) until feasibility is reached, and then improving the quality of this solution by using a standard LB or RINS approach.

\section{Computational Results}

In this section, we report on computational results comparing the proposed method with both the FP heuristic and the commercial software ILOG-Cplex 9.0.3. In our experiments, we used the "asymmetric" version of the local branching constraint (7), namely

$$
\Delta(x, \bar{x}):=\sum_{j \in \bar{S}}\left(1-x_{j}\right) .
$$


Indeed, as discussed in [9], this version of the constraint seems to be particularly suited for set covering problems where LB aims at finding solutions with a small binary support - which is precisely the case of interest in our context.

Our testbed is made up of 44 0-1 MIP instances from MIPLIB 2003 [1] and described in Table 1, plus an additional set of 39 hard 0-1 MIPs described in Table 2 and available, on request, from the second author. (The 0-1 MIPLIB instance stp3d was not considered since the computing time required for the first LP relaxation is larger than 1 hour, while 11 instances, namely fixnet6, markshare1, markshare2, mas74, mas76, modglob, pk1, pp08a, pp08aCUTS, set1ch and vpm2 have been removed because all tested algorithms found a feasible solution within $0.0 \mathrm{CPU}$ seconds.) The two tables report the name, total number of variables $(n)$, number of 0-1 variables $(|\mathcal{B}|)$, and number of constraints $(m)$ for each instance.

\begin{tabular}{|c|c|c|c|c|c|c|c|}
\hline Name & $n$ & $|\mathcal{B}|$ & $m$ & Name & $n$ & $|\mathcal{B}|$ & $m$ \\
\hline 10teams & 2025 & 1800 & $\overline{230}$ & nod011 & 10958 & 96 & 4480 \\
\hline A1C1S1 & 3648 & 192 & 3312 & todglob & 422 & 98 & 291 \\
\hline flow & 842 & 421 & 479 & nomentum1 & 5174 & 2349 & 42680 \\
\hline flow 4 & 2728 & 1364 & 1442 & net12 & 14115 & 1603 & 14021 \\
\hline ir04 & 8904 & 8904 & 823 & isrand_ipx & 6621 & 6620 & 735 \\
\hline r05 & 7195 & 7195 & 426 & lw04 & 87482 & 87482 & 36 \\
\hline ap6000 & 6000 & 6000 & 2176 & opt1217 & 769 & 768 & 64 \\
\hline $\operatorname{mip}$ & 13873 & 552 & 3202 & 56 & 2756 & 2756 & 755 \\
\hline danoint & 521 & 56 & 664 & pk1 & 86 & 55 & 45 \\
\hline $\mathrm{ds}$ & 67732 & 67732 & 656 & pp08a & 240 & 64 & 136 \\
\hline fast05 & 63009 & 63009 & 507 & pp08aCUTS & 240 & 64 & 246 \\
\hline fiber & 1298 & 1254 & 363 & protfold & 1835 & 1835 & 2112 \\
\hline fixn & 878 & 378 & 478 & qiu & 840 & 48 & 1192 \\
\hline glas & 322 & 302 & 396 & rd-rplusc-21 & 622 & 457 & 125899 \\
\hline harp & 2993 & 2993 & 112 & set1ch & 712 & 240 & 492 \\
\hline liu & 1156 & 1089 & 2178 & seymour & 1372 & 1372 & 4944 \\
\hline marl & 62 & 50 & 6 & p9 & 14101 & 14101 & 1761 \\
\hline marl & 74 & 60 & 7 & wath & 6805 & 6724 & 884 \\
\hline mast & 151 & 150 & 13 & $\mathrm{t} 1717$ & 73885 & 73885 & 551 \\
\hline $\operatorname{mas} 7$ & 151 & 150 & 12 & $\operatorname{tr} 12-30$ & 1080 & 360 & 750 \\
\hline $\operatorname{misc} 07$ & 260 & 259 & 212 & van & 12481 & 192 & 27331 \\
\hline mkc & 5325 & 5323 & 3411 & vpm2 & 378 & 168 & 234 \\
\hline
\end{tabular}

Table 1: The 44 0-1 MIP instances collected in MIPLIB 2003 [1]

The framework described in the previous section has been tested by using different starting solutions $\hat{x}$ provided by FP. In particular, we wanted to test the sensitivity of our modified LB algorithm with respect to the degree of infeasibility of the starting solution, as well as its capability for improving it. Thus, we executed the FP code for 0,10 and 100 iterations and passed to LB the integer (infeasible) solution $\hat{x}$ with minimum distance $\Phi\left(x^{*}, \hat{x}\right)$ from $P_{L}$. (The case with 0 iterations actually corresponds to starting from the solution of the continuous relaxation, rounded to the nearest integer.) The resulting three versions of the modified $\mathrm{LB}$ are called $\mathrm{LB}_{0}, \mathrm{LB}_{10}$, and $\mathrm{LB}_{100}$, respectively.

In our experiments, we avoided any parameter tuning-FP was implemented exactly as in [10], and for the modified LB code we used a time limit of $30 \mathrm{CPU}$ seconds for the exploration of each local-branching neighborhood. As to the value of the neighborhood-size parameter $k$ in LB, we implemented an adaptive procedure: at each neighborhood exploration, we try to reduce the number of violated constraints in the current solution by half, i.e., we set $k=\left\lfloor\left|T^{\prime}\right| / 2\right\rfloor$, where $\left|T^{\prime}\right|$ is the value of the current solution. (Since the support of the solution also takes into account non-artificial binary variables, when the number of violated constraints becomes less than 20 we fix $k=10$, i.e., we use the value suggested in [9] for the asymmetric version of the local branching constraint.). The motivation for this choice is that the number of violated constraints in an initial solution can be extremely large, in which case the use of a small value of $k$ would result in a very slow convergence. 


\begin{tabular}{|c|c|c|c|c|c|c|c|c|c|}
\hline Name & $n$ & $|\mathcal{B}|$ & $m$ & source & Name & $n$ & $|\mathcal{B}|$ & $m$ & source \\
\hline biella1 & 7328 & 6110 & 1203 & [9] & blp-ar98 & 16021 & 15806 & 1128 & [20] \\
\hline NSR8K & 38356 & 32040 & 6284 & {$[9]$} & blp-ic97 & 9845 & 9753 & 923 & [20] \\
\hline dc1c & 10039 & 8380 & 1649 & [8] & blp-ic98 & 13640 & 13550 & 717 & {$[20]$} \\
\hline dc1l & 37297 & 35638 & 1653 & [8] & blp-ir98 & 6097 & 6031 & 486 & [20] \\
\hline dolom1 & 11612 & 9720 & 1803 & [8] & CMS750_4 & 11697 & 7196 & 16381 & [18] \\
\hline siena1 & 13741 & 11775 & 2220 & [8] & berlin_5_8_0 & 1083 & 794 & 1532 & [18] \\
\hline trento1 & 7687 & 6415 & 1265 & [8] & railway_8_1_0 & 1796 & 1177 & 2527 & [18] \\
\hline rail507 & 63019 & 63009 & 509 & {$[9]$} & usAbbrv.8.25_70 & 2312 & 1681 & 3291 & [18] \\
\hline rail $2536 \mathrm{c}$ & 15293 & 15284 & 2539 & {$[9]$} & manpower1 & 10565 & 10564 & 25199 & [25] \\
\hline rail $2586 \mathrm{c}$ & 13226 & 13215 & 2589 & [9] & manpower2 & 10009 & 10008 & 23881 & [25] \\
\hline rail4284c & 21714 & 21705 & 4284 & [9] & manpower3 & 10009 & 10008 & 23915 & [25] \\
\hline rail4872c & 24656 & 24645 & 4875 & [9] & manpower3a & 10009 & 10008 & 23865 & [25] \\
\hline $\mathrm{A} 2 \mathrm{C} 1 \mathrm{~S} 1$ & 3648 & 192 & 3312 & [9] & manpower4 & 10009 & 10008 & 23914 & [25] \\
\hline B1C1S1 & 3872 & 288 & 3904 & {$[9]$} & manpower $4 a$ & 10009 & 10008 & 23866 & [25] \\
\hline B2C1S1 & 3872 & 288 & 3904 & [9] & $\overline{\mathrm{ljb} 2}$ & 771 & 681 & 1482 & {$[7]$} \\
\hline sp97ic & 12497 & 12497 & 1033 & {$[9]$} & ljb7 & 4163 & 3920 & 8133 & {$[7]$} \\
\hline sp98ar & 15085 & 15085 & 1435 & [9] & ljb9 & 4721 & 4460 & 9231 & {$[7]$} \\
\hline sp98ic & 10894 & 10894 & 825 & [9] & ljb10 & 5496 & 5196 & 10742 & {$[7]$} \\
\hline bg512142 & 792 & 240 & 1307 & {$[22]$} & ljb12 & 4913 & 4633 & 9596 & [7] \\
\hline
\end{tabular}

Table 2: The additional set of 39 0-1 MIP instances

All codes are written in ANSI C and use the ILOG-Cplex callable libraries. They are available, on request, from the second author. The three modified LB codes $\left(\mathrm{LB}_{0}, \mathrm{LB}_{10}\right.$, and $\left.\mathrm{LB}_{100}\right)$ are compared with FP and ILOG-Cplex 9.0.3 in Table 3 for the MIPLIB-2003 instances, and in Table 4 for the additional set of instances. Computing times are expressed in CPU seconds, and refer to a Pentium M 1.6 GHz notebook with 512 MByte of main memory. A time limit of 1,800 CPU seconds was provided for each instance with each algorithm and the computation was halted as soon as a first feasible solution was found.

For each instance, we report in both tables: for ILOG-Cplex, the number of nodes (nodes) needed to find an initial solution and the corresponding computing time (time); for FP, the number of iterations (FPit) and its computing time (time); for each of the three variants of LB, the computing time spent in the FP preprocessing phase (FP time), the initial number of violated constraints $(|T|)$, the number of LB iterations (LBit), and the overall computing time (time). Note that, we define an LB iteration as the exploration, generally within a time limit, of the neighborhood of the current solution. Moreover, the time reported is the sum of the time of the FP initialization plus the LB time, thus it can be larger than 1,800 CPU seconds. When one of the algorithms was not able to find a feasible solution in the given time limit, we wrote $\left(^{*}\right)$ in column "nodes" (for ILOG-Cplex) or "FPit" (for FP), or wrote $(\mu)$ in column "|T|" near the number of initial infeasible constraints (for LB), where $\mu$ is the number of violated constraints in the final solution.

As expected, the degree of infeasibility of the starting solution plays an important role in the LB methodsthe better the initial solution, the faster the method. In this view, the FP approach seems to fit particularly well in our context, in that it is able to provide very good solutions (as far as the degree of infeasibility is concerned) in very short computing times. Among the three LB implementations, $\mathrm{LB}_{0}$ was at least as fast as the other two in 40 cases, $\mathrm{LB}_{10}$ in 47 cases, and $\mathrm{LB}_{100}$ in 58 cases. Overall, $\mathrm{LB}_{100}$ qualifies as the most effective (and stable) of the three methods. (The figures above include the instances removed from Tables 3 and 4 because all algorithms required $0.0 \mathrm{CPU}$ seconds).

A comparison of ILOG-Cplex and $\mathrm{LB}_{100}$ shows that the latter is strictly faster in 44 cases, while the opposite holds in 21 cases. Moreover, ILOG-Cplex was not able to find any feasible solution (within the 1,800-second time limit) in 4 cases, whereas $\mathrm{LB}_{100}$ was unsuccessful 3 times. As expected, the quality of the initial ILOG-Cplex solution (not reported in the tables) is typically better than that provided by the LB methods. 


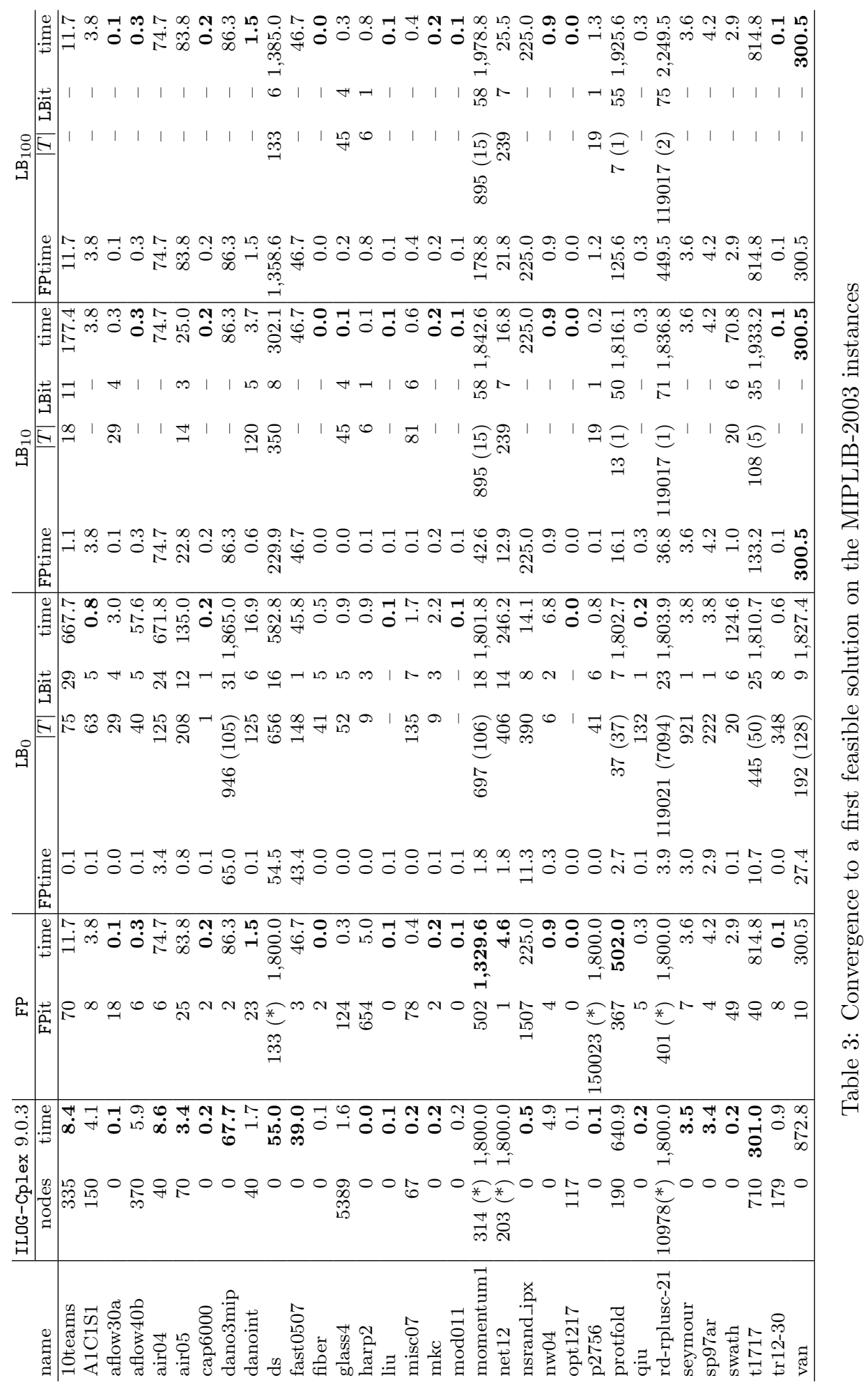




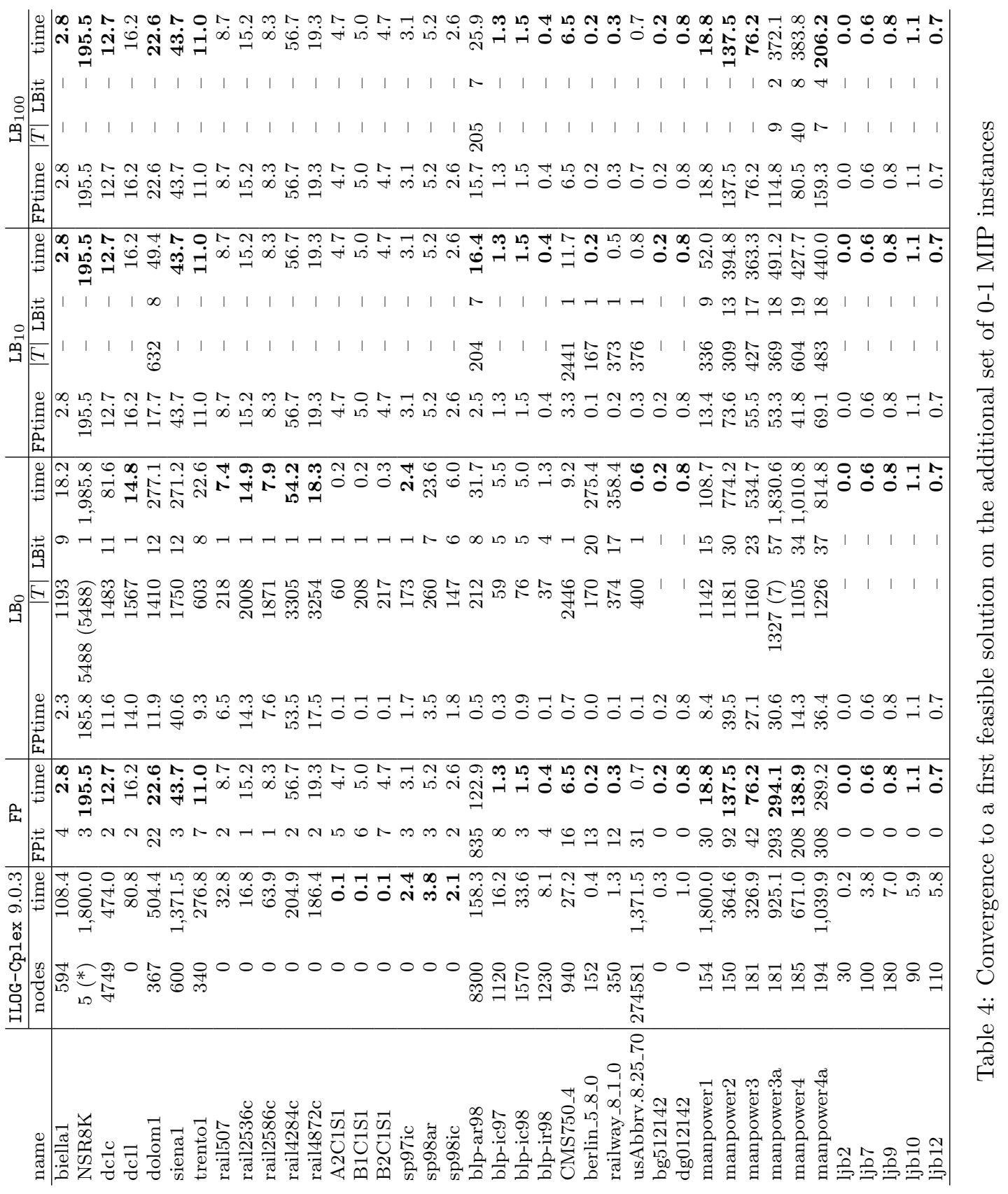




\section{Acknowledgements}

This work was partially supported by MIUR and CNR, Italy, and by the EU project "ADONET". Thanks are due to the anonymous referees for their helpful comments.

\section{References}

[1] T. Achterberg, T. Koch, A. Martin. The mixed integer programming library: MIPLIB 2003. http://miplib.zib.de.

[2] E. Amaldi, M.E. Pfetsch, L.E. Trotter, On the Maximum Feasible Subsystem Problem, IISs and IISHypergraphs. Mathematical Programming 95, 3, 533-554, 2003.

[3] E. Balas, S. Ceria, M. Dawande, F. Margot, G. Pataki. OCTANE: A New Heuristic For Pure 0-1 Programs. Operations Research 49, 207-225, 2001.

[4] E. Balas, C.H. Martin. Pivot-And-Complement: A Heuristic For 0-1 Programming. Management Science 26, 86-96, 1980.

[5] E. Balas, S. Schmieta, C. Wallace. Pivot and Shift-A Mixed Integer Programming Heuristic. Discrete Optimization 1, 3-12, 2004.

[6] J. Chinneck. Fast Heuristics for the Maximum Feasible Subsystem Problem. INFORMS Journal on Computing 13 (3), 210-223, 2001.

[7] E. Danna, E. Rothberg, C. Le Pape. Exploring relaxation induced neighborhoods to improve MIP solutions. Mathematical Programming 102, 71-90, 2005.

[8] Double-Click sas. Personal communication, 2001.

[9] M. Fischetti, A. Lodi. Local Branching. Mathematical Programming 98, 23-47, 2003.

[10] M. Fischetti, F. Glover, A. Lodi. The Feasibility Pump. Mathematical Programming 104, 91-104, 2005.

[11] M. Fischetti, C. Polo, M. Scantamburlo. A Local Branching Heuristic for Mixed-Integer Programs with 2-Level Variables. Networks 44, 2, 61-72, 2004.

[12] J. Gleesonand, J. Ryan. Identifying Minimally Infeasible Subsystems of Inequalities. ORSA Journal on Computing 2 (1), 61-63, 1990.

[13] F. Glover, M. Laguna. General Purpose Heuristics For Integer Programming: Part I. Journal of Heuristics 2, 343-358, 1997.

[14] F. Glover, M. Laguna. General Purpose Heuristics For Integer Programming: Part II. Journal of Heuristics 3, 161-179, 1997.

[15] F. Glover, M. Laguna. Tabu Search. Kluwer Academic Publisher, Boston, Dordrecht, London, 1997.

[16] P. Hansen, N. Mladenovíc, D. Urosevíc. Variable Neighborhood Search and Local Branching. Technical Report G-2004-54, Les Chahiers du GERAD, June 2004 (to appear in Computers and Operations Research, 2005).

[17] F. S. Hillier. Effcient Heuristic Procedures For Integer Linear Programming With An Interior. Operations Research 17, 600-637, 1969.

[18] G.W. Klau. Personal communication, 2002. 
[19] T. Ibaraki, T. Ohashi, H. Mine. A Heuristic Algorithm For Mixed-Integer Programming Problems. Mathematical Programming Study 2, 115-136, 1974.

[20] A. Løkketangen. Heuristics for 0-1 Mixed-Integer Programming. In P.M. Pardalos and M.G.C. Resende (ed.s) Handbook of Applied Optimization, Oxford University Press, 474-477, 2002.

[21] A. Løkketangen, F. Glover. Solving Zero/One Mixed Integer Programming Problems Using Tabu Search. European Journal of Operational Research 106, 624-658, 1998.

[22] A.J. Miller. Personal communication, 2003.

[23] N. Mladenovíc, P. Hansen. Variable Neighborhood Search. Computers 63 Operations Research 24, 1097-1100, 1997.

[24] M. Nediak, J. Eckstein. Pivot, Cut, and Dive: A Heuristic for 0-1 Mixed Integer Programming Research Report RRR 53-2001, RUTCOR, Rutgers University, October 2001.

[25] E. Rothberg. Personal communication, 2002. 